\title{
Prevalence and clinical profile of metabolic syndrome in longevity: study from Guangxi Zhuang Autonomous Region, China
}

\author{
Xianghua He', Wei Zhang ${ }^{1}$, Guofang Pang ${ }^{1}$, Yuan Lv ${ }^{1}$, Caiyou Hu ${ }^{1 *}$ (D) and Ze Yang ${ }^{2 *}$
}

\begin{abstract}
Background: Metabolic syndrome (MetS) was a risk factor for cardiovascular diseases, yet the prevalence of MetS among nonagenarians and centenarians was rarely reported. Here we investigated the prevalence of MetS and its components among nonagenarians and centenarians in our Zhuang population from Bama, Guangxi Zhuang Autonomous Region, China.

Method: In Bama area, there registered 881 individuals who lived more than 90 years old in 269,800 local residents and our study involved 307 long-lived participants and 486 local younger (35-68 years) persons, as controls. MetS was defined according to the revised National Cholesterol Education Program's Adult Treatment Panel III (NCEP ATPIII) criteria.

Results: The overall prevalence estimates of MetS among longevity group were $28.0 \%$ based on NCEP ATPIII criteria. The most common metabolic component was elevated blood pressure (61.1\%), followed by raised fasting glucose (39.1\%) and low high-density lipoprotein cholesterol (low HDL-C) (28.0\%). The prevalence of MetS and abdominal obesity in women (33.6\% and $22.1 \%$ respectively) was higher than that of men (19.8\% and $3.7 \%$ respectively) ( $\left.P_{\text {range }}<.001-0.019\right)$. Compared with controls, long-lived individuals were more likely to have two or more metabolic abnormalities ( $P_{\text {range }}<0.001$ ), and less likely to have zero or one metabolic abnormality $\left(P_{\text {range }}<0.001-0.020\right)$.
\end{abstract}

Conclusion: This study showed substantiality the prevalence and clinical profile of MetS in longevity population in Guangxi Zhuang Autonomous Region, China.

Keywords: Longevity, Metabolic syndrome, Chinese

\section{Background}

Individuals who live the longest are of broad interest to researchers in recent years. In 2010, there were 1,948,286 nonagenarians (people aged 90-99) and 35,934 centenarians (people aged 100 and more) in China [1]. As the population of China aging, the number of people aged 90 or more is expected to grow. Since persons who live into their 90 s and over 100 are a testament to longevity, studies about their unique characteristics will expand our knowledge on how to extend life expectancy. Bama County is

\footnotetext{
*Correspondence: cyhu.hua@163.com; Yang_ze@sina.com

'Department of Neurology, Jiangbin Hospital, No 85 Hedi Road, Nanning, Guangxi Zhuang Autonomous Region 530021, China

${ }^{2}$ The MOH Key Laboratory of Geriatrics, Beijing Hospital, National Center of Gerontology, No.1 Dahua Road, Dongdan, Beijing 100730, People's Republic of China
}

an autonomous county under the jurisdiction of Hechi than 90 years old [3].

Metabolic syndrome (MetS), a constellation of metabolic disorders including obesity, raised blood pressure, (raised triglycerides and lowered high, may be of special interest because of the increased with age $[4,5]$. Studies about longevity popualso revealed different types of metabolic disturbances [6-12], but that the results varied across different countries and ethnics. Study from Croatia 
suggesting that nonagenarians and centenarians had lower prevalence of overweight, obesity and lower blood pressure [6]. Instead, centenarians from Poland showed that mildly elevated blood pressure is a marker for better health status [7]. Recent study of familial longevity from China revealed decreased diastolic blood pressure but increased systolic blood pressure in centenarians [8]. Similar discrepancy can also be found among studies of lipid profile and longevity. Biological study for longevity demonstrated that centenarians and their offspring have significantly larger high-density lipoprotein (HDL) levels and particle sizes and low-density lipoprotein (LDL) levels compared with controls [9, 10]. However, other studies did not find significant association of HDL-C levels with centenarians $[11,12]$.

All of these studies indicated that individuals with longevity might have different metabolic phenotypes from those general individuals under different ethnic background. But no reports on these metabolic items research integrated were seen now yet. Furthermore, the prevalence of MetS increased in Chinese population aged 60-95 [13]. Thus we perform the study to investigate the prevalence and clinical profile of MetS in longevity in Guangxi Zhuang Autonomous Region, China.

\section{Methods}

\section{Study population}

The project is a cross-sectional study within the framework of the "Longevity and Health of Aging Population in Guangxi China (LHAPGC)" [14]. In this study, "longevity" subjects were classified as participants who had survived to age 90 years or more, with "unrelated younger controls" aged 35-68 years. A random sample of 793 individuals belonged to the Zhuang population from Bama (total population: 269,800) was recruited, including 307 long-lived individuals (256 nonagenarians and 51 centenarians) and 486 local and unrelated younger controls. Zhuang population is one of the largest ethnic groups in mainland China, second only to the Han population. The individuals with longevity included 226 women and 81 men (mean age: $95.06 \pm 4.91$ years and $94.60 \pm 4.09$ years old for women and men, respectively; range: $90-111$ years old). The control group comprised 185 women and 301 men (mean age: $47.98 \pm 4.07$ and $47.24 \pm 3.70$ years old for women and men, respectively; range: $35-68$ years old).

The survey was conducted using a uniform standardized protocol. All participants were examined by a senior physician and underwent extensive neuropsychological test as well as taking instrumental examination such as electrocardiogram and ultrasound examination. Individuals with longevity as well as controls were excluded if they had chronic disease such as malnutrition, hepatic disease, kidney disease and cancer. All controls refer to local and unrelated younger participants in general population.

The study was conducted according to the principles expressed in the Declaration of Helsinki. The Ethics Committee of Beijing Hospital, Ministry of Health approved the study protocol. Written informed consent was obtained from each of the participants.

\section{Measurements}

MetS was diagnosed as three or more of the following five factors as defined by the revised National Cholesterol Education Program's Adult Treatment Panel III (NCEP ATPIII) criteria for Asians (the American Heart Association and the National Heart, Lung, and Blood Institute (AHA/NHLBI) revised in 2005 [4], the same as the joint interim statement in 2009 [5]: (1) waist circumference $\geq 90 \mathrm{~cm}$ in males and $\geq 80 \mathrm{~cm}$ in females; (2) systolic blood pressure (SBP) $\geq 130 \mathrm{mmHg}$ or diastolic blood pressure (DBP) $\geq 85 \mathrm{mmHg}$ or taking antihypertensive drugs; (3) fasting blood glucose $\geq 5.6 \mathrm{mmol} / \mathrm{L}$ or taking drugs for diabetes; (4) triglycerides $\geq 1.7 \mathrm{mmol} / \mathrm{L}$ or taking antihyperlipidemic drugs; (5) HDL-C $<1.03 \mathrm{mmol} / \mathrm{L}$ in males and $<1.29 \mathrm{mmol} / \mathrm{L}$ in females or taking antihyperlipidemic drugs.

Body mass index (BMI) was determined as weight $(\mathrm{kg})$ divided by height $(\mathrm{m})$ squared. Waist circumference was measured at the mid-point between the lowermost rib and the iliac crest.

\section{Laboratory measurements}

Blood samples were collected after at least eight hours overnight fast for serum biochemistry, lipid profile, and plasma glucose in all participants. Clinical biomarkers including fasting plasma glucose (FPG), triglyceride (TG), and high-density lipoprotein cholesterol (HDL-C) were determined following standard laboratory procedures. Blood pressure was measured using a standard mercury sphygmomanometer on the right arm after at least $10 \mathrm{~min}$ of rest.

\section{Statistical analysis}

All statistical analyses were conducted using the SPSS 18.0 software package. One-Way ANOVA and a chisquared test were used to compare demographic and clinical data between longevity and controls. Age- and sex- adjusted were applied according to the 2010 population census of the people's republic of China from National Bureau of Statistics [1]. A two-tailed $P<0.05$ was considered statistically significant. 


\section{Results}

Demographic and metabolic characteristics in two groups Data for demographic characteristics were showed as numbers, and data for metabolic characteristics were presented as median and interquartile range (INR) in Table 1. Except for frequency of alcohol intake $(P=0.772)$, there were significant difference for frequency of cardiovascular disease, smoking status and sex ratio between the longevity group and the control one ( $\left.\mathrm{P}_{\text {range }}<.001\right)$. The study consisted of 793 participants with a female preponderance in the longevity group (Male: Female $=1: 2.79$ ). Generally, subjects in the longlived individuals cohort had significantly lower levels of height, body mass index (BMI), weight, waist circumference (WC), and TG than controls $\left(\mathrm{P}_{\text {range }}<.001-0.002\right)$, except for systolic blood pressure (SBP), diastolic blood pressure (DBP), and FPG ( $\left.\mathrm{P}_{\text {range }}<.001\right)$ (see Table 1 ). There was no difference of HDL-C level between two groups $(P=0.766)$.

\section{Overall prevalence of metabolic profile}

Table 2 showed the prevalence of MetS among the longevity of Zhuang Population from Guangxi Zhuang Autonomous Region. The prevalence of MetS was significantly higher in the longevity group $(28.0 \%)$ than it is in the local general control group (5.1\%) (0R:0.139, 95\%CI: $0.087-0.224, P<.001)$ regardless of the used criteria $\left(\mathrm{P}_{\text {range }}<.001\right)$ (see Table 2 ). The most common metabolic component in longevity individuals was high blood pressure (61.1\%), followed by raised fasting glucose (39.1\%) and lowHDL-C (28.0\%). In addition, the prevalence of high blood pressure (HBP) (0R:0.106,

Table 1 Demographic and metabolic characteristics of the study samples between longevity and controls

\begin{tabular}{|c|c|c|c|}
\hline Variables & Longevity $(N=307)$ & Control( $N=486)$ & $P$ \\
\hline Cardiovascular disease(N) & 17 & 0 & $<.001$ \\
\hline \multicolumn{4}{|l|}{ Smoking status(N) } \\
\hline Never & 295 & 420 & \multirow[t]{3}{*}{$<.001$} \\
\hline Former & 12 & 15 & \\
\hline Current & 0 & 51 & \\
\hline \multicolumn{4}{|c|}{ Cigarettes per day (current)(N) } \\
\hline$\leq 10$ & 0 & 35 & \multirow[t]{2}{*}{-} \\
\hline$\geq 11$ & 0 & 31 & \\
\hline \multicolumn{4}{|c|}{ Frequency of alcohol intake(N) } \\
\hline 1-3 times/month & 6 & 10 & \multirow[t]{4}{*}{0.772} \\
\hline 1-2 times/week & 23 & 44 & \\
\hline 3-4 times/week & 8 & 20 & \\
\hline Nearly 1 time/day & 5 & 6 & \\
\hline \multicolumn{4}{|l|}{$\operatorname{Drugs}(\mathrm{N})$} \\
\hline Antidyslipidemic & 0 & 0 & \multirow[t]{3}{*}{-} \\
\hline Antihypertensive & 2 & 0 & \\
\hline Antidiabetic & 0 & 0 & \\
\hline Gender(M/F)(N) & $81 / 226$ & $301 / 185$ & $<.001$ \\
\hline Age (Median(INR)) & $94(91-98)$ & $48(45-49)$ & $<.001$ \\
\hline Height (Median(INR)) & 145.0(140.0-151.0) & 164.5(158.0-169.5) & $<.001$ \\
\hline Weight (Median(INR)) & $38(34-45)$ & $66(58-72)$ & $<.001$ \\
\hline BMI (Median(INR)) & 18.35(16.41-20.93) & $24.11(22.57-26.00)$ & $<.001$ \\
\hline SBP (Median(INR)) & 146(132-160) & 120(110-130) & $<.001$ \\
\hline DBP (Median(INR)) & $80(72-90)$ & $76(70-84)$ & $<.001$ \\
\hline WC (Median(INR)) & $71.0(65.0-78.0)$ & $80.5(75.0-86.0)$ & $<.001$ \\
\hline FPG (Median(INR)) & $5.08(4.30-6.16)$ & $4.89(4.47-5.24)$ & $<.001$ \\
\hline TG (Median(INR)) & $1.08(0.77-1.55)$ & $1.52(1.22-1.86)$ & 0.002 \\
\hline HDL-C (Median(INR)) & $1.40(1.10-1.81)$ & $1.46(1.30-1.68)$ & 0.766 \\
\hline
\end{tabular}

INR interquartile range, $B M I$ body mass index, $S B P$ systolic blood pressure, $D B P$ diastolic blood pressure, WC waist circumference, $F P G$ fasting plasma glucose, $T G$ triglyceride, $H D L-C$ high-density lipoprotein cholesterol 
Table 2 Clinical metabolic characteristics between longevity and controls

\begin{tabular}{|c|c|c|c|c|c|c|c|}
\hline & \multirow{2}{*}{$\begin{array}{l}\text { Longevity } \\
n=307(100 \%)\end{array}$} & \multirow{2}{*}{$\begin{array}{l}\text { Control } \\
n=486(100 \%)\end{array}$} & \multirow[b]{2}{*}{$x^{2}$} & \multirow[b]{2}{*}{ OR } & \multicolumn{2}{|l|}{$95 \% \mathrm{Cl}$} & \multirow[b]{2}{*}{$P$} \\
\hline & & & & & Lower & $\overline{\text { Upper }}$ & \\
\hline$\overline{A O B}$ & $17.3 \%$ & $13.6 \%$ & 2.002 & 0.753 & 0.508 & 1.116 & 0.157 \\
\hline Elevated BP & $61.1 \%$ & $38.9 \%$ & 183.15 & 0.106 & 0.075 & 0.150 & $<.001$ \\
\hline RFG & $39.1 \%$ & $7.2 \%$ & 121.65 & 0.121 & 0.080 & 0.183 & $<.001$ \\
\hline hightG & $21.8 \%$ & $35.6 \%$ & 16.910 & 1.980 & 1.426 & 2.749 & $<.001$ \\
\hline lowHDL-C & $33.2 \%$ & $10.7 \%$ & 61.005 & 0.241 & 0.166 & 0.350 & $<.001$ \\
\hline MetS & $28.0 \%$ & $5.1 \%$ & 81.740 & 0.139 & 0.087 & 0.224 & $<.001$ \\
\hline
\end{tabular}

95\% Cl 95\% Confidence Interval, $A O B$ abdominal obesity, Elevated BP elevated blood pressure, RFG raised fasting glucose, highTG high triglyceride, low HDL-C low high-density lipoprotein cholesterol, MetS metabolic syndrome

95\%CI: $0.075-0.150, P<.001)$, raised fasting glucose (0R:0.121, 95\%CI: 0.080-0.183, $P<.001$ ), and lowHDLC (0R:0.241, 95\%CI: $0.166-0.350, P<.001)$ was significantly higher in long-lived individuals than it is in control subjects. On the contrary, the prevalence of highTG (0R:1.980, 95\%CI: 1.426-2.749, $P<.001$ ) was much lower in people with longevity than the controls (see Table 2). There was no difference of the abdominal obesity between longevity individuals and controls (0R:0.753, 95\%CI: 0.508-1.116, $P=0.157$ ) (see Table 2).

\section{Prevalence of metabolic profile in longevity}

Since numbers of male and female in longevity group are not equal, we compared the prevalence of clinical metabolic data among male and female participants. We did not find significant difference of age, height, weight, BMI, SBP, DBP, WC, FPG, TG and HDL-C among male and female long-lived individuals ( $P_{\text {range }}>0.05$ ).

Compared to males, the estimated odds of having MetS were 2.057 times higher in female longevity subjects (0R: 2.057, 95\%CI: 1.118-3.787, $P=0.019$ ) (see Table 3 ). At the same time, females were more likely to have abdominal obesity compared to males $(P<.001)$ (see Table 3). No significant difference of prevalence for elevated BP, raised fasting glucose, highTG, and lowHDL-C among male and female individuals aged 90+ years were noted in this study (see Table 3 ).

\section{Trend for frequency of metabolic abnormalities in two groups}

In this study, people aged 90+ have much higher prevalence of MetS than that of the controls (28.0\% vs. $5.1 \%)$ (See Table 2). For further analyzing the trend of MetS, we researched the prevalence of having zero, one, two, three, four, and five MetS components in the two groups. Table 4 showed that Compared with controls, long-lived individuals were more likely to have two or more components of MetS (for longevity: 36.2\% and $28.0 \%$ respectively; for controls: $21.2 \%$ and $5.1 \%$ respectively; $\mathrm{P}_{\text {range }}<0.001$ ), and less likely to have zero or one components of MetS (for longevity: $6.2 \%$ and $29.6 \%$ respectively; for controls: $32.1 \%$ and $41.6 \%$ respectively; $\left.\mathrm{P}_{\text {range }}<0.001-0.020\right)$.

\section{Discussion}

The present study provided the information on the overall prevalence estimates of MetS in longevity group been $28.0 \%$ based on NCEP ATPIII criteria. The most common metabolic component was high blood pressure, followed by raised fasting glucose and lowHDL-C. The prevalence of MetS and abdominal obesity in women was higher than that of men. No significant difference of metabolic components among longevity participants were found after stratified by gender. The prevalence of MetS among longevity individuals was significantly

Table 3 MetS and its components in longevity individuals stratified by gender

\begin{tabular}{|c|c|c|c|c|c|c|c|}
\hline & \multirow{2}{*}{$\begin{array}{l}\text { Male } \\
N=81(100 \%)\end{array}$} & \multirow{2}{*}{$\begin{array}{l}\text { Female } \\
N=226(100 \%)\end{array}$} & \multirow[b]{2}{*}{$x^{2}$} & \multirow[b]{2}{*}{ OR } & \multicolumn{2}{|l|}{$95 \% \mathrm{Cl}$} & \multirow[b]{2}{*}{$P$} \\
\hline & & & & & lower & upper & \\
\hline $\mathrm{AOB}$ & 3.70 & 22.10 & - & 7.386 & 2.236 & 24.405 & $<.001^{*}$ \\
\hline Elevated BP & 79.00 & 83.60 & 0.876 & 1.357 & 0.715 & 2.574 & 0.349 \\
\hline RFG & 43.20 & 37.60 & 0.785 & 0.792 & 0.473 & 1.327 & 0.376 \\
\hline highTG & 18.50 & 23.00 & 0.705 & 1.315 & 0.693 & 2.495 & 0.401 \\
\hline lowHDL-C & 27.20 & 35.00 & 1.641 & 1.441 & 0.823 & 2.525 & 0.200 \\
\hline MetS & 19.80 & 33.60 & 5.507 & 2.057 & 1.118 & 3.787 & 0.019 \\
\hline
\end{tabular}

${ }^{*}: P$ value according to Fisher's Exact Test

95\% Cl 95\% Confidence Interval, $A O B$ abdominal obesity, Elevated BP elevated blood pressure, RFG raised fasting glucose, highTG high triglyceride, low HDL-C low high-density lipoprotein cholesterol, MetS metabolic syndrome 
Table 4 Frequency of metabolic abnormalities in two groups

\begin{tabular}{|c|c|c|c|c|c|c|c|c|}
\hline \multirow[b]{2}{*}{ MetS components (N) } & \multicolumn{3}{|l|}{ Longevity } & \multicolumn{3}{|l|}{ Control } & \multirow[b]{2}{*}{$P$} & \multirow[b]{2}{*}{ OR } \\
\hline & Frequency & Percent & Mean \pm Sd. & Frequency & Percent & Mean \pm Sd. & & \\
\hline 0 & 19 & 6.2 & $95.21 \pm 4.662$ & 156 & 32.1 & $47.05 \pm 4.030$ & $<.001$ & $0.117-0.317$ \\
\hline 1 & 91 & 29.6 & $95.21 \pm 4.682$ & 202 & 41.6 & $47.53 \pm 3.341$ & 0.02 & $0.536-0.949$ \\
\hline 2 & 111 & 36.2 & $94.88 \pm 4.810$ & 103 & 21.2 & $47.79 \pm 4.144$ & 0.001 & $1.259-2.232$ \\
\hline 3 & 63 & 20.5 & $94.63 \pm 4.867$ & 22 & 4.5 & $49.36 \pm 5.141$ & $<.001$ & $2.733-7.519$ \\
\hline 4 & 19 & 6.2 & $94.68 \pm 4.372$ & 3 & 0.6 & $48.00 \pm 4.359$ & $<.001^{*}$ & $2.942-34.165$ \\
\hline 5 & 4 & 1.3 & $95.25 \pm 2.986$ & 0 & 0 & - & $0.023^{*}$ & - \\
\hline Total & 307 & 100 & $94.94 \pm 4.702$ & 486 & 100 & $47.52 \pm 3.859$ & - & - \\
\hline
\end{tabular}

${ }^{*} P$ value according to Fisher's Exact Test

Std standard deviation

higher than the local general control individuals. Interestingly, we found that compared with controls, longevous individuals were more likely to have two or more components of MetS, and less likely to have zero or one components of MetS.

To the best of our knowledge, rare studies examined the prevalence of MetS in longevity subjects. Studies from Sichuan Province, China, reported a prevalence rate of MetS with $9.3 \%$ to $10.8 \%$ in the total participants among individuals aged $90+$ years old $[15,16]$, which was lower than ours. However, another Chinese crosssectional study reported the prevalence rate of MetS was $50.4 \%$ in 2001 and $58.1 \%$ in 2010 among subjects aged 60-95 years [13], which was much higher than ours. The difference prevalence rate of MetS may be due to different ethnics and different human age stages. Further multicenter large-scale sample for longevity are needed to address this problem.

The most common metabolic component among longevity subjects in our Zhuang population was inconsistent with studies from other ethnic population $[17,18]$. For example, studies performed in Korea found that the most common component was high blood sugar levels, followed by elevated triglyceride levels and high blood pressure in males, and that elevated triglyceride levels, followed by high blood sugar levels and high blood pressure in females in a 66-year-old population [18]. In China, it has been shown that the most common component was elevated blood pressure, followed by central obesity and raised fasting glucose among Chinese aged 60 years or older[19]. Since the age for the participants in these studies were much younger than ours, it is possible that these inconsistent findings regarding different prevalence of metabolic components could be age differences. Another possible explanation may be explained by ethnic differences.

Females with longevity had higher prevalence of MetS than the male participants, which were consistent with several studies from different countries and ethnic population $[13,17,18]$. It is interesting that women had higher prevalence rates of MetS compared with men. According to the National Bureau of Statistics of China, the female/male ratio of longevity population were 2.02:1 (648,588 male nonagenarians, 1,299,698 female nonagenarians, 8852 male centenarians and 27,082 female centenarians in 2010) [1]. In our study, females also occupied a larger proportion (female/male: 2.79/1) in the longevity population. Thus, this group may be more representative of an ordinary group for longevious people, and they may be more sensitive to the risk factors from MetS than men. In addition, women who become postmenopausal had a significantly increased visceral abdominal fat $[20,21]$, accompanied by insulin resistance and hypertriglyceridemia, ultimately meeting the diagnosis of metabolic syndrome[22]. However, for females aged $90+$ years and for who had menopause for more than 40 years, it is hard to say hormonal regulation had an effect on their metabolic state. Additional consideration is required for sex differences between men and women among the oldest old.

In this study, women were more likely to have abdominal obesity than men in the longevity group. No significant difference of prevalence for high BP, dysglycemia, highTG, and lowHDL-C among male and female individuals aged $90+$ years were observed in this study. This result was inconsistent with other studies. For example, studies performed in China shown that the prevalence of TG, HDL-C, and WC among females is higher than the prevalence in males among Chinese aged 60-95 years old [19]. In Turkey, researchers found that older adult females had higher SBP, larger WC, and lowHDL-C than older males [23]. In Korea, prevalence of abdominal obesity and lowHDL-C in females were higher than males, while prevalence of highTG, high BP, and dysglycemia in males were higher than females [18]. The difference among different countries may be due to different ethnics and sample size. Multicenter Collaboration across different countries is needed to address this question. 
In our study, the high prevalence of MetS in longevity group could be linked to the observed higher prevalence of high BP, raised fasting glucose and lowHDL-C and lower prevalence of highTG compared to the control group. These findings were supported by previous studies which demonstrated nonagenarians and centenarians had higher prevalence of confirmed hypertension, diabetes mellitus as well as dyslipidemia [6-8, 24]. Moreover, a study investigating frailty and metabolic syndrome in a Chinese community sample showed that, in those aged 90 years and older, frailty was a significant risk for near-term death, regardless of the metabolic syndrome [16]. Furthermore, another cross-sectional study implicated that metabolic syndrome may be associated with better cognitive function among nonagenarians and centenarians [15]. Thus, it seemed that MetS was not a risk factor for the oldest old. However, it is well established that MetS is strongly related to increased incidence of cardiovascular events in people aged 60-95 $[13,25]$. One possible explanation of why MetS has a different effect among different stages of human beings might be because of "natural selection effect." It is possible that those with more severe MetS have died of cardiovascular diseases before reaching an older age. Another possible explanation is that MetS components are associated with better health status among the oldest old $[7,9,26]$. Specifically, low prevalence of highTG might contribute a lot to longevity in our study, as low TG level has been identified as a marker for human longevity $[26,27]$. Lastly, there was a possibility that traditional cardiovascular risk factors, such as an elevated cholesterol and hypertension, might do not automatically apply to the very old.

Moreover, we found that long-lived individuals had higher frequency for having two or more metabolic abnormalities and lower frequency for having zero or one metabolic abnormality than the controls, which was consistent with previous studies that prevalence of MetS increased with age $[13,28]$. More research is needed for the oldest old with MetS.

In our study, the control individuals had higher frequency of smoking than the longevity participants, which might explain the possibility for fewer controls having MetS, as smoking is a risk factor for younger people $[29,30]$.

However, our study has some limitations. Firstly, this was a cross-sectional study. Many variables were measured at a single time point and may be subject to conditions at the time of measurement. Since some of the study population had several risk factors including hyperlipidemia, we could not eliminate the possible effect of underlying diseases and medications used for these diseases. Secondly, the data came from a single region, which may limit generalizability. Thirdly, the sample size was not big enough in our study. Fourthly, the prevalence of various lipid and hematological parameters was based on a single assessment of blood, which may introduce a misclassification bias. Lastly, we have excluded Individuals with chronic disease such as malnutrition, hepatic disease, kidney disease and cancer, which might underestimate the prevalence of MetS in our population, especially in long-lived individuals. Multicenter collaboration in prospective research of prevalence for MetS in longevity group is needed to address these questions.

\section{Conclusions}

In conclusion, our study analyzed the prevalence of MetS and its components distribution in longevity individuals in Zhuang population. We also reported that there existed the markedly differences of MetS rates by sex-specific groups in the population, and that longlived individuals were more likely to have two or more metabolic abnormalities, and less likely to have zero or one metabolic abnormality than the controls.

\section{Abbreviations \\ 95\% Cl: 95\% Confidence Interval; AHA/NHLBI: American Heart Association and the National Heart, Lung, and Blood Institute; AOB: Abdominal obesity; BMI: Body mass index; DBP: Diastolic blood pressure; FPG: Fasting plasma glucose; HDL-C: High-density lipoprotein cholesterol; INR: Interquartile range; LDL-C: Low-density lipoprotein cholesterol; MetS: Metabolic syndrome; NCEP ATPIII: National Cholesterol Education Program's Adult Treatment Panel III; SBP: Systolic blood pressure; Sd: Standard deviation; TG: Triglyceride; WC: Waist circumference}

\section{Acknowledgements}

Our thanks go to all the patients, legal representatives and personnel of the geriatric wards for their participation. We would like to thank Professor Liang Sun for his assistance with statistical analysis.

\section{Funding}

This work was supported by the Natural Science Foundation of China $(81,460,203,81,400,790,81,241,082,81,061,120,527,81,370,445,81,472,408$, and 81,571,385); National Science and Technology Support Program, No. 2015BAI06B02; Key Program of Natural Science Foundation of Guangxi Province of China, No. 2014GXNSFDA118028; Guangxi Medical Health Appropriate Technology Research and Development Project, P. R. China. No S201402-1; Guangxi Science Research and Technology Development Project, China (Guikegong 1,355,005-6-2); Natural Science Foundation of Guangxi Province (Guikezi 0991198);Beijing Nova program (Z121107002512058);National Department Public Benefit Research Foundation by Ministry of Health P. R. China (201302008); and 12th 5 Year National Program from the Ministry of Scientific Technology (2012BAl10B01)

\section{Availability of data and materials}

The data for this research consists of in-person interview transcripts. Raw data cannot be publically released due to the risk the respondent confidentiality will be compromised.

\section{Authors' contributions}

X.H.H., W.Z., G.F.P., Y.L., Z.Y. and C.Y.H. were responsible for the study conception and design. X.H.H. collected and analyzed the data and wrote the paper. Z.Y. and C.Y.H. supervised the study. W.Z., G.F.P., and Y.L. commented on drafts of the manuscript. All authors read and approved the final manuscript. 


\section{Ethics approval and consent to participate}

The Ethics Committee of Beijing Hospital, Ministry of Health approved the study protocol. Written informed consent was obtained from each of the participants.

\section{Consent for publication}

Not applicable.

\section{Competing interests}

The authors declare that they have no competing interests.

\section{Publisher's Note}

Springer Nature remains neutral with regard to jurisdictional claims in published maps and institutional affiliations.

Received: 16 November 2016 Accepted: 6 July 2017

Published online: 31 July 2017

\section{References}

1. National Bureau of Statistics of China. Tabulation on the 2010 population census of the people's republic of China. 2011. http://www.stats.gov.cn/tjsj/ pcsj/rkpc/6rp/indexch.htm. Accessed 15 Mar 2017.

2. Gaikuang: Bamadiqingwang 2011. http://www.gxdqw.com/preview/ bmdqw/tiandi/201111/t20111103_10214.html. Accessed 06 June 2017.

3. BamaCounty: Baidu Baike 2013. http://baike.baidu.com/item/ \%E5\%B7\%B4\%E9\%A9\%AC\%E7\%91\%B6\%E6\%97\%8F\%E8\%87\%AA\% E6\%B2\%BB\%E5\%8E\%BF/7181053. Accessed 06 June 2017.

4. Grundy SM, Cleeman JI, Daniels SR, Donato KA, Eckel RH, Franklin BA, Gordon DJ, Krauss RM, Savage PJ, Smith SC Jr, et al. Diagnosis and management of the metabolic syndrome. An American Heart Association/ National Heart, Lung, and Blood Institute scientific statement. Executive summary. Cardiol Rev. 2005;13(6):322-7.

5. Alberti KG, Eckel RH, Grundy SM, Zimmet PZ, Cleeman JI, Donato KA, Fruchart JC, James WP, Loria CM, Smith SC Jr. Harmonizing the metabolic syndrome: a joint interim statement of the international diabetes federation task force on epidemiology and prevention; National Heart, Lung, and Blood Institute; American Heart Association; world heart federation; international atherosclerosis society; and International Association for the Study of obesity. Circulation. 2009;120(16):1640-5.

6. Masanovic M, Sogoric S, Kolcic I, Curic I, Smoljanovic A, Ramic S, Cala M, Polasek $\mathrm{O}$. The geographic patterns of the exceptional longevity in Croatia. Coll Antropol. 2009;33(Suppl 1):147-52.

7. Szewieczek J, Dulawa J, Francuz T, Legierska K, Hornik B, Wlodarczyk-Sporek I, Janusz-Jenczen M, Batko-Szwaczka A. Mildly elevated blood pressure is a marker for better health status in polish centenarians. Age (Dordr). 2015; 37(1):9738.

8. He YH, Pu SY, Xiao FH, Chen XQ, Yan DJ, Liu YW, Lin R, Liao XP, Yu Q, Yang $L Q$, et al. Improved lipids, diastolic pressure and kidney function are potential contributors to familial longevity: a study on 60 Chinese centenarian families. Sci Rep. 2016;6:21962.

9. Milman S, Atzmon G, Crandall J, Barzilai N. Phenotypes and genotypes of high density lipoprotein cholesterol in exceptional longevity. Curr Vasc Pharmacol. 2014;12(5):690-7.

10. Barzilai N, Atzmon G, Schechter C, Schaefer EJ, Cupples AL, Lipton R, Cheng S, Shuldiner AR. Unique lipoprotein phenotype and genotype associated with exceptional longevity. JAMA. 2003;290(15):2030-40.

11. Heijmans BT, Beekman M, Houwing-Duistermaat JJ, Cobain MR, Powell J, Blauw GJ, van der Ouderaa F, Westendorp RG, Slagboom PE. Lipoprotein particle profiles mark familial and sporadic human longevity. PLoS Med. 2006;3(12)::495.

12. Gong YY, Xie L, Zhou WP, Zhang YP, Gong YY, Xie L, Lian SG, Yang J, Wang $X Y$, Yang Z, et al. Glucose and lipid profile of a long-lived rural Han Chinese population and their families in southwest China. J Am Geriatr Soc. 2009; 57(3):567-8

13. Liu M, Wang J, Jiang B, Sun D, Wu L, Yang S, Wang Y, Li X, He Y. Increasing prevalence of metabolic syndrome in a Chinese elderly population: 20012010. PLoS One. 2013;8(6):e66233.

14. Feng J, Zhang J, Liu M, Wan G, Qi K, Zheng C, Lv Z, Hu C, Zeng Y, Gregory $\mathrm{SG}$, et al. Association of mtDNA haplogroup $\mathrm{F}$ with healthy longevity in the female Chuang population, China. Exp Gerontol. 2011;46(12):987-93.
15. Luo L, Yang M, Hao Q, Yue J, Dong B. Cross-sectional study examining the association between metabolic syndrome and cognitive function among the oldest old. J Am Med Dir Assoc. 2013;14(2):105-8.

16. Hao Q, Song X, Yang M, Dong B, Rockwood K. Understanding risk in the oldest old: frailty and the metabolic syndrome in a Chinese community sample aged 90+ years. J Nutr Health Aging. 2016;20(1):82-8.

17. Kuk JL, Ardern $\mathrm{Cl}$. Age and sex differences in the clustering of metabolic syndrome factors: association with mortality risk. Diabetes Care. 2010;33(11): $2457-61$.

18. Lee S, Ko Y, Kwak C, Yim ES. Gender differences in metabolic syndrome components among the Korean 66-year-old population with metabolic syndrome. BMC Geriatr. 2016;16:27.

19. He Y, Jiang B, Wang J, Feng K, Chang Q, Fan L, Li X, Hu FB. Prevalence of the metabolic syndrome and its relation to cardiovascular disease in an elderly Chinese population. J Am Coll Cardiol. 2006;47(8):1588-94.

20. Stefanska A, Bergmann K, Sypniewska G. Metabolic syndrome and menopause: Pathophysiology, clinical and diagnostic significance. Adv Clin Chem. 2015;72:1-75.

21. Park JK, Lim YH, Kim KS, Kim SG, Kim JH, Lim HG, Shin J. Body fat distribution after menopause and cardiovascular disease risk factors: Korean National Health and nutrition examination survey 2010. J Women's Health (Larchmt). 2013:22(7):587-94.

22. Pradhan AD. Sex differences in the metabolic syndrome: implications for cardiovascular health in women. Clin Chem. 2014;60(1):44-52

23. Akbulut G, Koksal E, Bilici S, Acar Tek N, Yildiran H, Karadag MG, Sanlier N. Metabolic syndrome (MS) in elderly: a cross sectional survey. Arch Gerontol Geriatr. 2011;53(3):e263-6.

24. Motta M, Bennati E, Capri M, Ferlito L, Malaguarnera M. Diabetes mellitus in the extreme longevity. Exp Gerontol. 2008;43(2):102-5.

25. Vinluan CM, Zreikat HH, Levy JR, Cheang Kl. Comparison of different metabolic syndrome definitions and risks of incident cardiovascular events in the elderly. Metabolism. 2012;61(3):302-9.

26. Mazzotti DR, Guindalini C, Moraes WA, Andersen ML, Cendoroglo MS, Ramos LR, Tufik S. Human longevity is associated with regular sleep patterns, maintenance of slow wave sleep, and favorable lipid profile. Frontiers Aging neurosci. 2014;6:134.

27. Gonzalez-Covarrubias V. Lipidomics in longevity and healthy aging Biogerontology. 2013;14(6):663-72.

28. Mahabaleshwarkar R, Taylor YJ, Spencer MD, Mohanan S. Prevalence of metabolic syndrome in a large integrated health care system in North Carolina. N C Med J. 2016;77(3):168-74.

29. Xi B, He D, Hu Y, Zhou D. Prevalence of metabolic syndrome and its influencing factors among the Chinese adults: the China health and nutrition survey in 2009. Prev Med. 2013;57(6):867-71.

30. Slagter SN, van Vliet-Ostaptchouk JV, Vonk JM, Boezen HM, Dullaart RP, Kobold AC, Feskens EJ, van Beek AP, van der Klauw MM, Wolffenbuttel BH. Associations between smoking, components of metabolic syndrome and lipoprotein particle size. BMC Med. 2013;11:195.

\section{Submit your next manuscript to BioMed Central} and we will help you at every step:

- We accept pre-submission inquiries

- Our selector tool helps you to find the most relevant journal

- We provide round the clock customer support

- Convenient online submission

- Thorough peer review

- Inclusion in PubMed and all major indexing services

- Maximum visibility for your research

Submit your manuscript at www.biomedcentral.com/submit
) Biomed Central 